\title{
Male-specific phosphorylated SR proteins in adult flies of the Mediterranean Fruitfly Ceratitis capitata
}

\author{
Giuseppe Saccone ${ }^{1 *}$, Christos Louis ${ }^{2}$, Hongyou Zhang ${ }^{3 *}$, Valeria Petrella ${ }^{1}$, Manuela Di Natale ${ }^{1}$, Maria Perri ${ }^{1}$, \\ Marco Salvemini ${ }^{1}$
}

\begin{abstract}
Alternative splicing is a widely used mechanism of gene regulation in sex determination pathways of Insects. In species from orders as distant as Diptera, Hymenoptera and Coleoptera, female differentiation relies on the activities of conserved splicing regulators, TRA and TRA-2, promoting female-specific expression of the global effector doublesex (dsx). Less understood is to what extent post-translational modifications of splicing regulators plays a role in this pathway. In Drosophila melanogaster phosphorylation of TRA, TRA-2 and the general RBP1 factor by the LAMMER kinase doa (darkener of apricot) is required for proper female sex determination. To explore whether this is a general feature of the pathway we examined sex-specific differences in phosphorylation levels of SR splicing factors in the dipteran species D. melanogaster, Ceratitis capitata (Medfly) and Musca domestica (Housefly). We found a distinct and reproducible pattern of male-specific phosphorylation on protein extracts enriched for SR proteins in C. capitata suggesting that differential phosphorylation may also contribute to the regulation of sex-specific splicing in the Medfly.
\end{abstract}

\section{Introduction}

Alternative splicing (AS) can be regulated in tissue-specific and/or stage-specific manners and can be responsive to signaling cues [1]. Regulation of alternative splicing is achieved through the interaction of trans-acting splicing regulator complexes with the global splicing machinery. Gene-specific AS is regulated by differential expression, degradation, phosphorylation and intracellular localization of splicing factors [2]. Each splicing factor can have antagonistic effects on the alternative splicing outputs on few specific genes or on coordinated group of genes [3,4].

Serine/arginine-rich (SR) proteins are splicing factors involved in the regulation of several developmental processes, including sex determination in Insects, which share a RRM-type RNA binding domain and are rich in serine/arginine residues [5-9]. Phosphorylation of the

\footnotetext{
* Correspondence: giuseppe.saccone@unina.it; hongyu.zhang@mail.hzau.edu. cn

'Department of Biology, University of Naples Federico II, 80134, Naples, Italy ${ }^{3}$ State Key Laboratory of Agricultural Microbiology and Institute of Urban and Horticultural Pests, College of Plant Science and Technology, Huazhong Agricultural University, Wuhan 430070, People's Republic of China

Full list of author information is available at the end of the article
}

serine/arginine repeats provokes a structural change that alters the functional state of these splicing regulators, thereby affecting developmental choices [10-13]. Protein phosphorylation plays an essential role in the regulation of sex-specific splicing in Drosophila [10]. The LAMMER kinase DOA is responsible for phosphorylating the SR protein RBP1 and most likely other two splicing factors: TRA-2 (SR protein) and the female-specific TRA (serine arginine rich, lacking the RMM domain), which promote female-specific splicing of doublesex. DOA phosphorylates also a second SR major protein, DX16, which is the Drosophila ortholog of the human SFRS7 9G8 SR protein [14].

In the Mediterranean fruit fly C. capitata and in the housefly $M$. domestica, the mechanism of sex determination of Drosophila is partially conserved [15-19]. In these species, TRA and TRA-2, which are related to SR proteins, function as splicing regulators of doublesex $(d s x)$ and fruitless (fru) pre-mRNAs, promoting their female-specific expression and, ultimately, female sexual differentiation [16,20-25]. Orthologs of the Drosophila tra gene in different insect orders appear to be ON/OFF




master switch genes for sex determination and are female-specifically expressed through alternative splicing [26]. Furthermore, except for Drosophila, tra is also able to regulate itself, promoting female-specific splicing of its own pre-mRNA to maintain continuous expression of TRA activity $[27,28]$. It is still unknown, though, how the male-specific splicing of tra, which leads to truncated, non-functional TRA isoforms, is regulated by the male determining factors in C. capitata and $M$. domestica $[25,29,30]$. Furthermore it is not yet clear if and how many downstream genes promoting sexual differentiation are also being controlled, during development and in different tissues, by sex-specific alternative splicing in Drosophila and other insect species [31-35].

To investigate if sex-specific SR phosphorylation takes place in adult dipteran flies, we used a commercially available mouse monoclonal antibody (mAb104), which surprisingly recognizes a highly conserved phosphoepitope shared by several major SR proteins (6-9) in metazoans, including vertebrates [36,37]. The SR family comprises at least 7 distinct major proteins in Drosophila and 9 in Homo sapiens; they all share the mAb104-phosphoepitope, which is also conserved in plants [38-41]. Interestingly, this antibody cross-reacts also with Drosophila TRA and TRA-2 baculovirus-expressed recombinant proteins in HeLa extracts, possibly owing to their phosphorylated SR domains promoted by human cell kinases [5]. We searched for sex-specific differences in phosphorylation of major SR splicing factors at adult stages of flies in the three distantly related dipteran species mentioned above (D. melanogaster, C. capitata and $M$. domestica), which are phylogenetically distant 130 million of years (mya), with C. capitata and Drosophila more closely related (120 mya).

\section{Results and discussion}

To search for sex-specific differences in the phosphorylation of major SR splicing factors in D. melanogaster, $C$. capitata and $M$. domestica, proteins were extracted from male and female adult tissues. We enriched for SR proteins following a two-step protocol as described in [41]. In Figure 1 whole staining of SR protein enriched extracts as well as immunoblots with the mAb104 antibody are depicted. In all samples we recovered a pattern of enriched SR proteins similar in both males and females (Figure 1A, B and C). Following quantitation of the supernatant proteins and of the soluble proteins after magnesium precipitation from the last purification step, SDS-PAGE separations were performed in parallel duplicates, with one stained with Coomassie Blue (to control for equal loading) and a second one blotted onto a filter. The transfer of proteins extracted from each sex was controlled by reversible Ponceau staining, which is a validated method to assess both equal gel loading and protein transfer [42,43] (Additional file 1 Figure S1). No sex-specific differences were evident. The filters were used for immunostaing and, on the contrary, showed some sex-specific differences. In Drosophila adult flies, the mAb104 antibody detects 6-8 polypeptides of molecular weights similar to those previously observed in Drosophila Kc cell line [37]. However, the relative amounts appear different in males and females (odd numbers: males; even numbers: females). For instance, the $95 \mathrm{kDa}$ signal is significantly stronger in males, while the $75 \mathrm{kDa}$ signal is more prominent in females (Figure 1D, lanes 7-8). In $M$. domestica four major phophorylated SR proteins were detected with comparable levels in both sexes (Figure 1E, Figure lanes 7-8). In two immunoblots with samples isolated from two different biological replicates of adult $C$. capitata flies, we observed that phosphorylated polypeptides were highly enriched only in males (Figure 1, lanes 7 of blots in $\mathrm{F}$ and $\mathrm{G})$. Two of the six major SR antigens detected $(\sim 75 \mathrm{kDa}$ and $\sim 30 \mathrm{kDa})$ can also be seen at much lower levels in the female samples in one of the 2 blots (Figure 1, blot in F, lanes 8 ). As only $0.04 \%$ of cell proteins are SR proteins [39] and the mAb104 can detect them only by $\mathrm{Mg}^{++}$enrichment, we propose that the male-specific C. capitata SR antigens correspond most likely to phosphorylated SR proteins expressed in most of the fly tissues rather then small tissues (such as for example the male germ line).

When samples were treated with a phosphatase prior to immunoblotting, four of the six bands detected in male extracts disappeared (Figure $1 \mathrm{H}$, lane $\mathrm{P}+$ ). The ones that persisted even after phosphatase treatment could also be detected in the female sample $(\sim 75 \mathrm{kDa}$ and $\sim 30 \mathrm{kDa}$ not shown). These signals probably result from mAb104 epitopes which were resistant to phosphatase treatment. This data suggests that the male-specific pattern observed in the C. capitata mAb104 immunoblots is due to male-specific phosphorylation for at least some of the SR proteins.

The absent (or strongly reduced) mAb104 signal in females could be due to absence of protein phosphorylation or to a lower expression of these SR proteins and corresponding genes. As a first step to investigate this question, we investigated by digital differential expression analysis (DGE) in adult $C$. capitata males and females using available RNA-seq data sets. The male and female reads were combined together and de novo assembled using Trinity software. We obtained 188,625 assembled transcripts with an N50 value of 1693 bp (data not shown). Using this data set six C. capitata SR orthologous proteins were identified by TBLASTN analysis and found to be highly conserved with the respect of the six Drosophila SR polypeptides (CcB52, CcRBP1, CcSC35, CcSRP54, CcX16 and CcASF/SF2, showing 


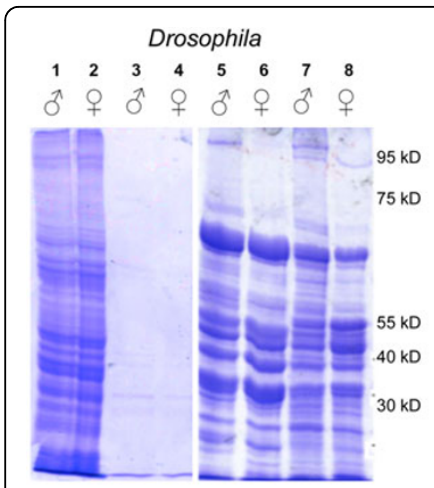

A



D

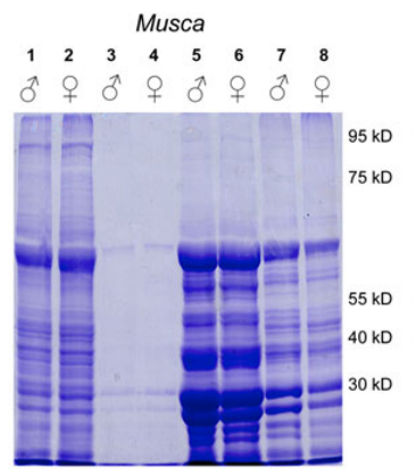

B

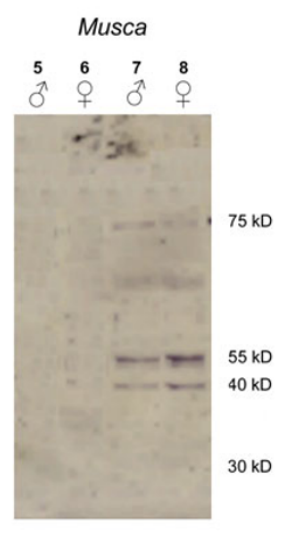

E

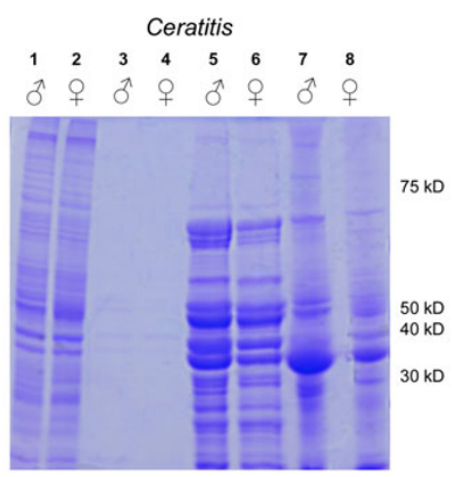

C



$\mathbf{F}$

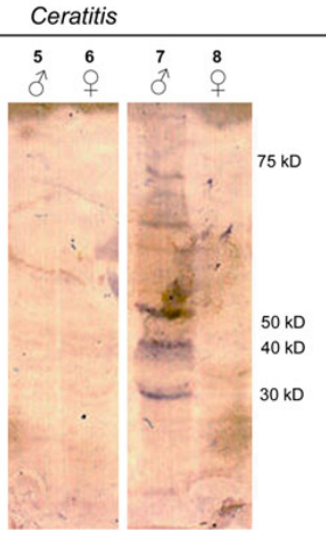

G

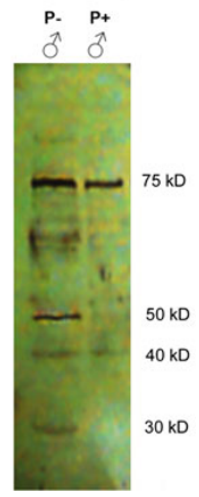

H

Figure 1 SDS-PAGE electropherograms (A-B-C) and immunoblots (D-E-F-G-H) produced with protein extracts from adult males and females of Drosophila, $\boldsymbol{M}$. domestica and C. capitata. 1) total lysate of males; 2) total lysate of females; 3) $90 \%$ ammonium sulfate precipitation supernatant of males; 4) $90 \%$ ammonium sulfate precipitation supernatant of females; 5) $\mathrm{Mg}^{++}$supernatant of males; 6) $\mathrm{Mg}^{++}$ supernatant of females; 7) $\mathrm{Mg}^{++}$pellets of males; 8) $\mathrm{Mg}^{++}$pellets of females; $\mathrm{P}-$ ) $\mathrm{Mg}^{++}$pellets of $\mathrm{C}$. capitata males without phosphatase treatment; $\mathrm{P}+) \mathrm{Mg}^{++}$pellets of $\mathrm{C}$. capitata males with phosphatase treatment. After transfer to nitrocellulose, blots were incubated with either mAb104 or no primary antibody (as a control; data not shown).

respectively $88 \%, 95 \%, 98 \%, 92 \%, 74 \%$ and $84 \%$ amino acid identity when compared to Drosophila). Male and female reads were then used separately to compute gene expression levels in both sexes (Additional file 2 - Table S1). We used the male-specific beta-tubulin and the female-specific $f$ st genes of Ceratitis as a preliminary DGE controls and found that the former is exclusively expressed in the male sex while the latter gene is only expressed in females. Although we have no replicates for this DGE analysis and, hence, we cannot measure the sex bias, this first control data is consistent with what was expected, suggesting that we could answer to the question whether SR-encoding mRNAs are present or not in females. When we used the six Ceratitis SR genes for DGE, no one showed absence of expression in females. We have choosen CcB52 for further analysis, we performed an RT-PCR, using two pair of primers and we got cDNA fragments in both sexes of adult flies. These data suggests that the observed male-specific phosphorylation patterns are likely due to increased phosphorylation rather than to higher transcription in males or reduced transcription in females.

In metazoans SR phosphorylation is mainly controlled by a serine-threonine kinase specific for the SR domain, which is therefore named SR protein-specific kinase 1 or SRPK1 [45]. In addition, the Cdc2-like kinase (CLK) family has also been implicated in SR protein phosphorylation events [46]. We therefore investigated whether SR-specific kinases are expressed only in C. capitata males. We identified two putative Ceratitis SRPK1related kinases and expression was observed in the two sexes by DGE analysis. A similar result was obtained for the Drosophila CLK kinase darkener of apricot (doa) ortholog in C. capitata which seems to produce mRNAs also in the adult females. The Drosophila doa gene expresses female-specific isoforms which may be involved in sex-specific SR phosphorylation events $[47,48]$. Further investigations would be needed to clarify if there are also alternative isoforms for some of 
these genes in C. capitata, which could have been differentially expressed in the two sexes.

\section{Conclusions}

In conclusion, we show that in two distantly related dipteran species, such as Drosophila and the housefly, the phosphorylation pattern of SR proteins detected by the mAb104 in adult flies seems to be non sex-specific. In contrast in C. capitata adult flies the SR phosphorylation mAb104 pattern of six proteins is almost exclusively male-specific or strongly enriched in this sex. The DGE data showed that in $C$. capitata the six SR encoding genes are transcribed and most likely translated also in the female adult flies. Based on our study we can only speculate that 1) the 6 Ceratitis SR proteins are transcribed and expressed in both sexes to perform basal alternative splicing regulations, related to their deep evolutionary metazoan conservation and 2) that the malespecific mAb104-detected SR phosphorylated proteins could have additional functions, possibly related to maleness, and to the control of sex-specific or sex-biased gene splicing $[49,50]$. As Ceratitis, Musca and Drosophila show a conservation of tra and $d s x$ sex-specific splicing regulation, but only Ceratitis show male-specific SR phoshorylated proteins, we propose that these splicing factors are involved in regulatory events either upstream or downstream to the tra $>$ dsx regulatory module.

We think that the observation of male-specific phosphorylated SR proteins in Ceratitis male flies is a preliminary necessary premise to provoke interest and to start further in silico and functional analysis also in other Tephritidae. Future experiments including SR proteins extraction from dissected $X Y$ fly body parts and from sexed embryos/ larvae will help to understand where and when the male-specific SR phosphorylation pattern is present, although we presently think that these SR antigens are present in most of the tissues of the adult male fly. Immunoprecipitation and sequencing will help to find further confirmation and information about identity of the SR antigens and related potential isoforms.

\section{Materials and methods Immunoblotting}

3-4 gr of flies were used for each protein sample and the final $\mathrm{Mg}^{++}$pellet (4th purification step) was resuspended in solution and entirely loaded in one gel lane. During the purification procedure an aliquot (10 microliters) from each of three different steps was saved for gel electrophoresis analyses. Tissues were ground to a fine powder in liquid nitrogen, using a mortar and pestle, and transferred to $30 \mathrm{ml}$ of the isolation buffer $(65 \mathrm{mM} \mathrm{KCl}, 15 \mathrm{mM}$ $\mathrm{NaCl}, 10 \mathrm{mM}$ HEPES at pH 7.6, $10 \mathrm{mM} \mathrm{Na} \mathrm{N}_{2}$ EDTA, $5 \mathrm{mM}$ DTT, $5 \mathrm{mM}$ potassium fluoride (KF), $5 \mathrm{mM}$ p-glycerophosphate, $0.2 \mathrm{mM}$ PMSF, and $2 \mathrm{~g} / \mathrm{ml}$ of aprotinin). The samples were sonicated and centrifuged to eliminate debris; from the supernatant a first aliquot of total proteins was saved from each sample for gel analysis (Figure 1, AB-C, lanes 1-2). The supernatant was used for a $65 \%$ (2 hr of stirring at $4^{\circ} \mathrm{C}$, and centrifugation at $8000 \mathrm{~g}$ for $20 \mathrm{~min}$ ) and then a $90 \%$ ammonium sulfate precipitations $(2 \mathrm{hr}$ of stirring at $4^{\circ} \mathrm{C}$ and centrifugation at $10,000 \mathrm{~g}$ at $4^{\circ} \mathrm{C}$ for 1 hr). A second aliquot (proteins from the sup 90\%) from the supernatant of the centrifuged $90 \%$ ammonium sulfate precipitation was saved for analyses (Figure 1, A-B-C, lanes 3-4). The pellets were resuspended in 200 microl of dialysis buffer $(65 \mathrm{mM} \mathrm{Kcl}, 15 \mathrm{mM} \mathrm{NaCl}, 10 \mathrm{mM}$ HEPES at pH 7.6, $1 \mathrm{mM} \mathrm{Na}{ }_{2}$ EDTA, $2 \mathrm{mM}$ DTT, $5 \mathrm{mM} \mathrm{KF}, 5$ $\mathrm{mM}$ p-glycerophosphate, and $0.2 \mathrm{mM}$ PMSF), dialyzed against three changes of $300 \mathrm{ml}$ of dialysis buffer over the course of $9 \mathrm{hr}$. The dialysate was recovered and stored at $-80^{\circ} \mathrm{C}$. The dialysate samples were thawed and centrifuged for $15 \mathrm{~min}$ at $13,000 \mathrm{~g}$. Supernatants were transferred to clean tubes, and $\mathrm{MgCl}_{2}$ was added to $20 \mathrm{mM}$. After a 1-hr incubation on ice, tubes were centrifuged at $13,000 \mathrm{~g}$ for $30 \mathrm{~min}$. After removal of the supernatants, from which samples were saved for gel analyses (Figure 1, A-B-C, lanes 5-6), the pellets were washed with 200 microliters of $20 \mathrm{mM} \mathrm{MgCl}$ dialysis buffer and resuspended in 20 microliters of $5 \%$ glycerol buffer D [41]. Sample buffer was added to the various samples obtained from the purification procedure and the proteins were separated by SDS-10\% PAGE (Figure 1, A-B-C, lanes 7-8). Similarly to the observations of Zahler et al. [41], the complexity of the protein samples throughout the 4 purification steps, was gradually reduced from step 1 to step 4, leading approximately to $\mathrm{Mg}^{++}$pellets containing 1-2 dozens prominent polypeptides visible by blue Coomassie stain in the 3 species. However, the observed grade of purification of the SR proteins (lanes 7-8) is lower than expected, being the electrophoretic pattern more complex if compared to the one described by Roth et al. and by Zahler et al. $[36,41]$. This could be due to the fact that in our study entire animals were used as starting samples, which are expected to be more complex compared to cell lines or dissected tissues in which it has been previously estimated that at least $0.04 \%$ of cell proteins are SR proteins [41]. However, most of the visible polypeptides in lanes 7 and 8 in the blue comassie are not detected by the mAb104 in the corresponding immunoblots (D-E-F-G).

\section{De novo assembly and digital expression analysis of medfly adult sexed transcriptomes}

Illumina RNA-seq raw reads were downloaded from NCBI SRA homepage (0-6 days old adult Medfly males: SRX272878; 0-6 days old adult Medfly females: SRX272877). The male and female paired end (PE) reads were combined together and de novo assembled using Trinity software (release 2012-10-05) [51] on the 
ALAN Server at the Department of Biology of the University of Naples Federico II (24 cores, 192 GB of memory). Trinity was run on the PE reads with the fixed default k-mer size of 25, minimum contig length of 200, maximum length expected between fragment pairs of 500, 22 CPUs, and a butterfly HeapSpace of $20 \mathrm{~GB}$. We obtained 188,625 assembled transcripts with an N50 value of $1693 \mathrm{bp}$. This adult data set was searched by TBLASTN analysis using as queries the $D$. melanogaster protein sequences of six SR genes (FlyBase Acc. Numbers: $\mathrm{B} 52=\mathrm{FBpp} 0300515, \mathrm{RBP} 1=\mathrm{FBpp} 0081754, \mathrm{SC} 35=$ FBpp0088838, SRP45= FBpp0079471, X16= FBpp0078996, SF2 $=$ FBpp0082724) and of ten Serine/Threonine kinase genes $(\mathrm{SRPK}=\mathrm{FBpp} 0303478$; $\operatorname{srpk79D}=\mathrm{FBpp} 0297551$; Doa $=$ FBpp0289029) and the $C$. capitata orthologs were identified (showing in C. capitata respectively $96 \%, 94 \%$, $97 \%, 97 \%, 99 \%, 81 \%, 88 \%, 84 \%, 71 \%$ and $91 \%$ of aa identity; in case of multiple isoforms the longest one was selected as representative). Male and female reads were then used separately to compute gene expression levels in both sexes, using FPKM metric, by the RSEM software [52], the Bowtie aligner [53] and the edgeR software [54], as implemented in the Trinity software package with default parameters. The C. capitata male-specifically expressed beta tubulin gene (Ccbeta2t) [55] the femalespecifically expressed $f s t$ gene [56] were used to assess the lack of contaminations of one sex with the other in the assembled adult transcriptomes.

\section{Additional material}

\section{Additional file 1: Figure S1 Reversible red-ponceau staining of} filters, used subsequently for mAb104 immuno-reactions. Figure 1 Ponceau staining of blots from SDS page electrophoresis of SR protein extracts of adult males and females of Drosophila (D), M. domestica (E) and C. capitata ( $F$ and G). 5) $\mathrm{Mg}^{++}$supernatant of males; 6) $\mathrm{Mg}^{++}$ supernatant of females; 7) $\mathrm{Mg}^{++}$pellets of males; 8$) \mathrm{Mg}^{++}$pellets of females; $\mathrm{P}-) \mathrm{Mg}^{++}$pellets of $\mathrm{C}$. capitata males without phosphatase treatment; $\mathrm{P}+) \mathrm{Mg}^{++}$pellets of $\mathrm{C}$. capitata males with phosphatase treatment.

Additional file 2: Table S1 Transcriptome analysis of SR-protein and Serine/Threonine kinase encoding genes in adult $C$. capitata. Expression levels for Medfly males $(X Y)$ and females $(X X)$ are reported using FPKM metric (Fragments Per Kilobase Of Exon Per Million Fragments Mapped). $\log F C=\log _{2}$ of fold change values; $\log C P M=\log _{2}$ of counts-per-million of mapped reads; $F D R=$ false discovery rate.

\section{Competing interests}

The authors declare that they have no competing interests.

\section{Authors' contributions}

GS, CL, MS wrote the paper. MD, MP, VP performed molecular experiments. MS performed bioinformatic and DGE analyses. $\mathrm{CL}$ and $\mathrm{HZ}$ supported the research with materials and technical ideas. MS. GS conceived the concept of the study, supervised and coordinated experiments. Read and approved the final manuscript: $G S, C L, Z H, V P, M D, M P, M S$,

\section{Acknowledgements}

We thank Dr. D. Bopp for providing the Musca adult samples and for his critical inputs on the manuscript. We with to greatly thank the retired Professor M. Lancieri, M. and Professor Emeritus L. C. Polito for their material and conceptual supports. This work was supported by a PRIN 2008 grant to GS and by China Agriculture Research System (No.CARS-27) and the Fundamental Research Funds for the Central Universities (No. 2014PY005) to HZ.

This research benefited from discussions at the International Atomic Energy Agency funded meetings of the Coordinated Research Project,

"Development and Evaluation of Improved Strains of Insect Pests for SIT" This article has been published as part of BMC Genetics Volume 15 Supplement 2, 2014: Development and evaluation of improved strains of insect pests for SIT. The full contents of the supplement are available online at http://www.biomedcentral.com/bmcgenet/supplements/15/S2.

Publication of this supplement was funded by the International Atomic Energy Agency. The peer review process for articles published in this supplement was overseen by the Supplement Editors in accordance with BioMed Central's peer review guidelines for supplements. The Supplement Editors declare that they have no competing interests.

\section{Authors' details}

'Department of Biology, University of Naples Federico II, 80134, Naples, Italy. ${ }^{2}$ Institute of Molecular Biology and Biotechnology, Foundation of Research and Technology-Hellas, GR - 70013 Heraklion, Crete, Greece. ${ }^{3}$ State Key Laboratory of Agricultural Microbiology and Institute of Urban and Horticultural Pests, College of Plant Science and Technology, Huazhong Agricultural University, Wuhan 430070, People's Republic of China.

Published: 1 December 2014

\section{References}

1. Braunschweig U, Gueroussov S, Plocik, et al: Dynamic integration of splicing within gene regulatory pathways. Cell 2013, 152:1252-1269.

2. Breig O, Baklouti F: Proteasome-mediated proteolysis of SRSF5 splicing factor intriguingly co-occurs with SRSF5 mRNA upregulation during late erythroid differentiation. PLOS ONE 2013, 8:e59137.

3. Calarco AJ, Superina S, O'Hanlon, et al: Regulation of vertebrate nervous system alternative splicing and development by an SR-related protein. Cell 2009, 138:898-910.

4. Blanchette M, Green RE, Brenner SE, Rio DC: Global analysis of positive and negative pre-mRNA splicing regulators in Drosophila. Genes Dev 2005, 19:1306-1314.

5. Long CJ, Caceres FJ: The SR protein family of splicing factors: master regulators of gene expression. Biochem J 2009, 417:15-27.

6. Nagaraju J, Saccone G: How is sex determined in insects? An epilogue. J Genet 2010, 89:389-390

7. Nagaraju J, Saccone G: How is sex determined in insects? Preface. J Genet 2010, 89:269-270.

8. Nagoshi NR, McKeown M, Burtis, et al: The control of alternative splicing at genes regulating sexual differentiation in D. melanogaster. Cell 1988, 53:229-236

9. Tian M, Maniatis T: Positive control of pre-mRNA splicing in vitro. Science 1992, 256:237-240

10. Du C, McGuffin EM, Dauwalder, et al: Protein phosphorylation plays an essential role in the regulation of alternative splicing and sex determination in Drosophila. Mol Cell 1998, 2:741-750.

11. Shepard JP, Hertel JK: The SR protein family. Genome Biol 2009, 10:242.

12. Hertel $S$, Tertre LA, Jockel, et al: Quantification of the heat wave effect on cause-specific mortality in Essen, Germany. Eur J Epidemiol 2009, 24:407-414.

13. Zhou Z, Fu D $\times$ : Regulation of splicing by SR proteins and SR proteinspecific kinases. Chromosoma 2013, 122:191-207.

14. Wan Y, Sun M, Wang, et al: DX16 is a novel SR protein phosphorylated by DOA. Mol Cell Biochem 2008, 307:177-183.

15. Graham P, Penn KJ, Schedl, et al: Masters change, slaves remain. Bioessays 2003, 25:1-4.

16. Hediger M, Henggeler $C$, Meier, et al: Molecular characterization of the key switch $f$ provides a basis for understanding the rapid divergence of the sex-determining pathway in the housefly. Genetics 2010, 184:155-170. 
17. Pane A, Salvemini M, Bovi D, et al: The transformer gene in Ceratitis capitata provides a genetic basis for selecting and remembering the sexual fate. Development 2002, 129:3715-3725.

18. Salz KH: Sex determination in insects: a binary decision based on alternative splicing. Curr Opin Genet Dev 2011, 21:395-400.

19. Sanchez L: Sex-determining mechanisms in insects. Int J Dev Biol 2008, 52:837-856.

20. Burghardt G, Hediger M, Siegenthaler, et al: The transformer2 gene in Musca domestica is required for selecting and maintaining the female pathway of development. Dev Genes Evol 2005, 215:165-176.

21. Hediger M, Burghardt $G$, Siegenthaler, et al: Sex determination in Drosophila melanogaster and Musca domestica converges at the level of the terminal regulator doublesex. Dev Genes Evol 2004, 214:29-42.

22. Meier N, Kappeli CS, Niessen H, et al: Genetic control of courtship behavior in the housefly: evidence for a conserved bifurcation of the sex-determining pathway. PLOS ONE 2013, 8:e62476.

23. Saccone G, Salvemini M, Pane, et al: Masculinization of XX Drosophila transgenic flies expressing the Ceratitis capitata DoublesexM isoform. Int J Dev Biol 2008, 52:1051-1057.

24. Salvemini $M$, Polito C, Saccone, et al: fruitless alternative splicing and sex behaviour in insects: an ancient and unforgettable love story? J Genet 2010, 89:287-299.

25. Salvemini $M$, Robertson $M$, Aronson, et al: Ceratitis capitata transformer-2 gene is required to establish and maintain the autoregulation of Cctra, the master gene for female sex determination. Int J Dev Biol 2009, 53:109-120.

26. Verhulst CEL, Van de Zande, Beukeboom LW: Insect sex determination: it all evolves around transformer. Curr Opin Genet Dev 2010, 20(4):376-83.

27. Bopp D, Saccone G, Beye, et al: Sex Determination in Insects: Variations on a Common Theme. Sex Dev 2013.

28. Saccone G, Salvemini M, Polito, et al: The transformer gene of Ceratitis capitata: a paradigm for a conserved epigenetic master regulator of sex determination in insects. Genetica 2011, 139:99-111.

29. Hediger $M$, Niessen M, Muller-Navia, et al: Distribution of heterochromatin on the mitotic chromosomes of Musca domestica L. in relation to the activity of male-determining factors. Chromosoma 1998, 107:267-271.

30. Willhoeft $U$, Franz $\mathrm{G}$ : Identification of the sex-determining region of the Ceratitis capitata Y chromosome by deletion mapping. Genetics 1996, 144:737-745

31. Clough E, Oliver B: Genomics of sex determination in Drosophila. Brief Funct Genomics 2012, 11:387-394.

32. Luo H, Dearolf RC: The JAK/STAT pathway and Drosophila development. Bioessays 2001, 23:1138-1147.

33. Ranz MJ, Castillo-Davis IC, Meiklejohn, et al: Sex-dependent gene expression and evolution of the Drosophila transcriptome. Science 2003 300:1742-1745.

34. Shukla NJ, Palli RS: Doublesex target genes in the red flour beetle, Tribolium castaneum. Sci Rep 2012, 2:948.

35. Telonis-Scott M, Kopp A, Wayne, et al: Sex-specific splicing in Drosophila: widespread occurrence, tissue specificity and evolutionary conservation. Genetics 2009, 181:421-434.

36. Roth BM, Murphy C, Gall, et al: A monoclonal antibody that recognizes a phosphorylated epitope stains lampbrush chromosome loops and small granules in the amphibian germinal vesicle. J Cell Biol 1990, 111:2217-2223.

37. Roth BM, Zahler MA, Stolk, et al: A conserved family of nuclear phosphoproteins localized to sites of polymerase II transcription. J Cell Biol 1991, 115:587-596.

38. Neugebauer MK, Stolk AJ, Roth, et al: A conserved epitope on a subset of SR proteins defines a larger family of Pre-mRNA splicing factors. J Cell Biol 1995, 129:899-908.

39. Zahler MA, Lane SW, Stolk, et al: SR proteins: a conserved family of premRNA splicing factors. Genes Dev 1992, 6:837-847.

40. Zahler MA, Neugebauer MK, Lane, et al: Distinct functions of SR proteins in alternative pre-mRNA splicing. Science 1993, 260:219-222.

41. Zahler MA, Neugebauer MK, Stolk, et al: Human SR proteins and isolation of a cDNA encoding SRp75. Mol Cell Biol 1993, 13:4023-4028.

42. Gilda JE, Gomes AV: Stain-Free total protein staining is a superior loading control to $\beta$-actin for Western blots. Anal Biochem 2013, 15:3.
43. Romero-Calvo I, Ocon B, Martinez-Moya, et al: Reversible Ponceau staining as a loading control alternative to actin in Western blots. Anal Biochem 2010, 401:318-320.

44. Vorbruggen $\mathrm{G}$, Onel $\mathrm{S}$, Jackle, et al: Localized expression of the Drosophila gene Dxl6, a novel member of the serine/arginine rich (SR) family of splicing factors. Mech Dev 2000, 90:309-312.

45. Gui FJ, Tronchere $\mathrm{H}$, Chandler, et al: Purification and characterization of a kinase specific for the serine- and arginine-rich pre-mRNA splicing factors. Proc Natl Acad Sci USA 1994, 91:10824-10828.

46. Aubol EB, Plocinik MR, Hagopian, et al: Partitioning RS domain phosphorylation in an SR protein through the CLK and SRPK protein kinases. J Mol Biol 2013, 425:2894-2909.

47. Rabinow L, Samson LM: The role of the Drosophila LAMMER protein kinase DOA in somatic sex determination. J Genet 2010, 89:271-277.

48. Rabinow L: CLK / LAMMER kinases. Heidelberg: Springer; 2012, 431-441.

49. Carvalho BA, Koerich BL, Clark, et al: Origin and evolution of $Y$ chromosomes: Drosophila tales. Trends Genet 2009, 25:270-277.

50. Vicoso B, Bachtrog D: Reversal of an ancient sex chromosome to an autosome in Drosophila. Nature 2013, 499:332-335.

51. Grabherr GM, Haas JB, Yassour, et al: Full-length transcriptome assembly from RNA-Seq data without a reference genome. Nat Biotechnol 2011, 29:644-652.

52. Li B, Dewey NC: RSEM: accurate transcript quantification from RNA-Seq data with or without a reference genome. BMC Bioinformatics 2011, $12: 323$.

53. Langmead B, Trapnell C, Pop, et al: Ultrafast and memory-efficient alignment of short DNA sequences to the human genome. Genome Biol 2009, 10:R25.

54. Robinson DM, McCarthy JD, Smyth, et al: edgeR: a Bioconductor package for differential expression analysis of digital gene expression data. Bioinformatics 2010, 26:139-140.

55. Scolari F, Schetelig MF, Bertin S, et al: Fluorescent sperm marking to improve the fight against the pest insect Ceratitis capitata (Wiedemann; Diptera: Tephritidae). N Biotechnol 2008, 25:76-84.

56. Ciolfi S, Filippis dT, Torti, et al: Molecular characterization and chromosomal localization of female-specific genes from the Mediterranean fruit fly Ceratitis capitata (Diptera: Tephritidae). Genome 2005, 48:139-144.

doi:10.1186/1471-2156-15-S2-S6

Cite this article as: Saccone et al:: Male-specific phosphorylated SR proteins in adult flies of the Mediterranean Fruitfly Ceratitis capitata. BMC Genetics 2014 15(Suppl 2):S6.

\section{Submit your next manuscript to BioMed Central and take full advantage of:}

- Convenient online submission

- Thorough peer review

- No space constraints or color figure charges

- Immediate publication on acceptance

- Inclusion in PubMed, CAS, Scopus and Google Scholar

- Research which is freely available for redistribution

Submit your manuscript at www.biomedcentral.com/submit
C Biomed Central 